\title{
Von der Vergangenheit, Gegenwart und Zukunft des Strafvollzugs und der Straftäterbehandlung
}

\author{
Klaus-Peter Dahle ${ }^{1}$ \\ Online publiziert: 22. Januar 2020 \\ (c) Der/die Autor(en) 2020
}

Das Gefängnis hat seit jeher den Auftrag, auf den Gefangenen einzuwirken und Veränderungsprozesse anzustoßen, mit dem Ziel, ihn zu motivieren und in die Lage zu versetzen, nach dem Ende der Gefangenschaft ein Leben ohne weitere Straftaten zu führen. Seitdem die Idee aufkam, zu diesem Zweck auch therapeutische Mittel einzusetzen, hat der damit entstandene Behandlungsgedanke eine streitbare Geschichte erlebt. Gestritten wurde über die grundsätzliche Legitimation, strafgefangene Menschen ohne deren Auftrag und Genehmigung überhaupt therapieren zu dürfen. Gestritten wurde über den Ressourcenaufwand, denn ein behandlungsorientierter Vollzug erfordert mehr als bloße Sicherung und Grundversorgung. Gestritten wurde weiterhin über die mit der therapeutischen Ausgestaltung des Gefängnisses vermeintlich einhergehende Milde eines zu „weichen“ Strafvollzugs, wodurch andere Strafzwecke - namentlich die Abschreckung - ausgehöhlt würden. Gestritten wurde schließlich über die grundsätzliche Erfolgsaussicht der Idee, Straftäter durch Behandlung von weiteren Straftaten abzuhalten.

Im Verlauf der 1990er-Jahre hatte sich dennoch ein moderater Behandlungsoptimismus verbreitet. In seinem Gefolge erweiterten sich hierzulande sukzessive die Aufgaben an den Justizvollzug. Einerseits stellten veränderte Zielgruppen neue Herausforderungen dar: zunehmend Gefangene aus unterschiedlichsten Kulturen, Gefangene aus organisierten Clanstrukturen, radikalisierte Gefangene; die Liste ließe sich fortführen. Andererseits erteilte der Gesetzgeber den Einrichtungen immer mehr und immer schwierigere Behandlungsaufträge, da die gesetzliche Indikation für eine Sozialtherapie in vielen Bundesländern nicht mehr nur Sexualstraftäter betrifft, sondern im Sinne des Risikoprinzips auf weitere Hochrisikogruppen ausgeweitet wurde. Aber auch sicherungsverwahrte Menschen und die von der

Prof. Dr. Klaus-Peter Dahle

klauspeter.dahle@uni-hildesheim.de

1 Institut für Psychologie, Universität Hildesheim, Hildesheim, Deutschland
Maßregel bedrohten Gefangenen haben mittlerweile einen gesetzlichen Anspruch auf eine maßgeschneiderte Behandlung. Es mehren sich die Anzeichen, dass der Justizvollzug an Grenzen stoßen könnte.

Es scheint deshalb angebracht innezuhalten, den gegenwärtigen Istzustand des nach Erziehungs- und Behandlungsgesichtspunkten orientierten Justizvollzugs und die Bedarfe seiner Klientel zu diagnostizieren und mögliche Wege zu erkunden, wie es weitergehen könnte. Dies ist das Anliegen des vorliegenden Bands 1 der Zeitschrift im neuen Jahrzehnt. Es ist ein volles Heft geworden, mit zunächst mehreren programmatischen Beiträgen, die den Status quo aus unterschiedlichen Perspektiven beleuchten und Überlegungen für weitere Entwicklungsmöglichkeiten anstellen. Den Anfang macht der Vorschlag einer niedersächsischen Arbeitsgruppe, neben den bisherigen Behandlungsansätzen in der Vollzugsforschung und Vollzugspraxis verstärkt entwicklungspsychologische Theorien, Methoden und Befunde zu berücksichtigen, da es sich bei der Entwicklungspsychologie um denjenigen Wissenschaftszweig handele, der sich grundsätzlich mit Veränderungsprozessen des Menschen über die Lebensspanne und mit den Voraussetzungen für Entwicklung beschäftige. Es folgt ein Beitrag des neuseeländischen Klinikers und Wissenschaftlers Tony Ward mit einem Plädoyer für mehr Theorieentwicklung und konzeptuelle Grundlagenarbeit in der Straftäterbehandlung. Demnach böte nur ein fundiertes Verständnis der Hintergründe kriminellen Verhaltens und der Voraussetzungen für Veränderung, so die Argumentation, ein hinreichendes Fundament für eine erfolgversprechende Rehabilitation von Rechtsbrechern. Der Erlanger Kriminologe und Kriminalpsychologe Friedrich Lösel analysiert aus einer empirischen Perspektive die bisherigen Entwicklungspfade der Straftäterbehandlung und ihre Erträgnisse und Befunde im Hinblick auf die zentrale Frage: „What works?“. Aus diesen Analysen leitet er 12 Vorschläge ab, wie die Behandlungs- und Vollzugsforschung aus Sicht der empirischen Erfahrungswissenschaft fortentwickelt werden könne. Der Beitrag des Schweizer Psychologen Bernd Borchard wirbt um das Züricher Modell einer ,deliktorien- 
tierten Therapie“. Dieses sei demnach geeignet, sowohl die Risikoorientierung des „What-works“-Ansatzes effizienter Straftäterbehandlung als auch die Ressourcenorientierung des „Desistance“-Ansatzes fruchtbar zu integrieren. Eine zu einseitige Deliktfokussierung in der Täterarbeit kritisiert hingegen der Berliner forensische Psychiater und Mitherausgeber der Zeitschrift Hans-Ludwig Kröber in seinem Beitrag zur rehabilitativen, ressourcenorientierten Therapie mit Straffälligen. Eine Fokussierung auf die Deliktarbeit in der Straftäterbehandlung lenke den Blick demnach einseitig auf Vermeidungsziele für Handlungen, die der Täter ohnehin selten zeige, und lege ihn auf die Rolle des Rechtsbrechers fest. Er plädiert demgegenüber für eine stärker holistische Grundhaltung unter Einbezug der Ressourcen des Täters und mit dem Ziel der Förderung seiner Resilienz.

Es ist somit ein streitbares Heft geworden mit durchaus kontroversen Beiträgen. Aber es findet/finden sich nicht nur Programmatisches, sondern auch empirische Beiträge rund um das Kernthema. So widmet sich der Beitrag einer Berliner Arbeitsgruppe jungen Gefangenen des Jugendstrafvollzugs, die behördlicherseits als Intensivtäter klassifiziert wurden, und untersucht deren Weg durch den Jugendvollzug und durch die Sozialtherapie. Eine weitere Berliner Arbeitsgruppe widmet sich der Suchtbehandlung unter den Bedingungen einer Justizvollzugsanstalt und plädiert im Ergebnis für eine viel stärkere Verzahnung intra- und extramuraler Behandlungsangebote. Der Beitrag des niedersächsischen kriminologischen Dienstes analysiert Entscheidungsprozesse bei der Indikationsstellung für eine Sozialtherapie und schlägt einen spezifischen Ansatz vor, solche Prozesse systematisch zu untersuchen. Den Abschluss bildet eine Arbeit aus der Kriminologischen Zentralstelle, die mit ihren Jahreserhebungen zur Sozialtherapie in Deutschland auf mittlerweile 23 Erhebungsjahre zurückblickt. Der Beitrag analysiert die Entwicklungen aus dieser Zeit und zeigt aktuelle Trends auf.
Natürlich finden sich auch die gewohnten Rubriken im Heft: Blitzlicht, Journal Club und Kongresskalender. Ich wünsche den Lesern also eine erkenntnisreiche, spannende und auch unterhaltsame Lektüre. Auf eine kleine Änderung bleibt aber noch hinzuweisen. Wie Sie vielleicht bemerkt haben, ist der neuseeländische Beitrag von Tony Ward zwar mit deutschem Titel und deutscher Zusammenfassung versehen, aber in englischer Sprache publiziert. Zwar bleibt die Forensische Psychiatrie Psychologie Kriminologie im Grundsatz eine deutschsprachige Zeitschrift. Die Herausgeber haben sich jedoch entschieden, zukünftig herausragende Arbeiten ausländischer Autoren auch in englischer Sprache zur Publikation anzunehmen, um deren Sichtbarkeit und Verbreitungsgrad in der FPPK zu erhöhen.

\section{Klaus-Peter Dahle}

Funding Open Access funding provided by Projekt DEAL.

Interessenkonflikt K.-P. Dahle gibt an, dass kein Interessenkonflikt besteht.

Open Access Dieser Artikel wird unter der Creative Commons Namensnennung 4.0 International Lizenz veröffentlicht, welche die Nutzung, Vervielfältigung, Bearbeitung, Verbreitung und Wiedergabe in jeglichem Medium und Format erlaubt, sofern Sie den/die ursprünglichen Autor(en) und die Quelle ordnungsgemäß nennen, einen Link zur Creative Commons Lizenz beifügen und angeben, ob Änderungen vorgenommen wurden.

Die in diesem Artikel enthaltenen Bilder und sonstiges Drittmaterial unterliegen ebenfalls der genannten Creative Commons Lizenz, sofern sich aus der Abbildungslegende nichts anderes ergibt. Sofern das betreffende Material nicht unter der genannten Creative Commons Lizenz steht und die betreffende Handlung nicht nach gesetzlichen Vorschriften erlaubt ist, ist für die oben aufgeführten Weiterverwendungen des Materials die Einwilligung des jeweiligen Rechteinhabers einzuholen.

Weitere Details zur Lizenz entnehmen Sie bitte der Lizenzinformation auf http://creativecommons.org/licenses/by/4.0/deed.de. 